$\stackrel{N / N}{=}$

Global Journals Inc.

(2)

GLOBAL JOURNAL OF MEDICAL RESEARCH: G

VETERINARY SCIENCE AND VETERINARY MEDICINE

Volume 20 Issue 2 Version 1.0 Year 2020

Type: Double Blind Peer Reviewed International Research Journal

\title{
Presence of Equine Infectious Anemia in work Horses inSan Andrés Itzapa, Chimaltenango, Guatemala
}

By García-García, Sandy Stephanie, Chávez-López, Juan José

\& Villatoro-Chacón, Daniela Mariel

Universidad de San Carlos de Guatemala

Abstract- Equine infectious anemia (IEA) is a worldwide distribution disease caused by a lentivirus associated withthe presence of transmitting vectors of the gender Tabanus and equines by producing anemiaandconsequently hypoxia that produces a multi-organ deterioration. The presence of AIE in the working equine of San Andrés Itzapa, Chimaltenango was evaluated. This in order to assess the presence of the disease since this group of equines is part of the food security of the community. 100 blood samples were analyzed using the gel agar immunodifusion test. The presence of the disease was found in $8 \%$ of the sampled animals. The prevalence was higher in females than in males. The meanage of the horses sampled was 5 years. The mean bodyconditionof the study population was 2 . The presence of the disease in the study population should be evaluated and considered as a starting point for the implementation of control and prevention measuresgiven the vector spread of the disease. -age and the impacton the community due to the zootechnical function of the sampled animals.

Keywords: anemia, equine, immunodiffusion.

GJMR-G Classification: NLMC Code: WC 900

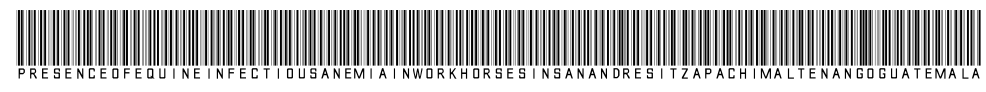

Strictly as per the compliance and regulations of:

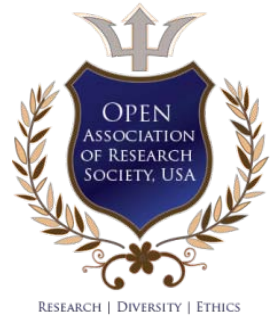

(C) 2020. García-García, Sandy Stephanie, Chávez-López, Juan José \& Villatoro-Chacón, Daniela Mariel. This is a research/review paper, distributed under the terms of the Creative Commons Attribution-Noncommercial 3.0 Unported License http://creativecommons.org/licenses/by-nc/3.0/), permitting all non-commercial use, distribution, and reproduction in any medium, provided the original work is properly cited. 


\title{
Presence of Equine Infectious Anemia in work Horses in San Andrés Itzapa, Chimaltenango, Guatemala
}

\author{
Presencia De Anemia Infecciosa Equina En Caballos De Trabajo En San Andrés \\ Itzapa, Chimaltenango, Guatemala
}

\author{
García-García, Sandy Stephanie ${ }^{\alpha}$, Chávez-López, Juan José ${ }^{\sigma}$ \& Villatoro-Chacón, Daniela Mariel ${ }^{\circ}$
}

Resumen- La anemia infecciosa equina (AIE) es una enfermedad de distribución mundial causada por un lentivirus asociado a la presencia de vectores transmisoresdel género Tabanus y Stomoxys. Esta enfermedad tiene un impacto en la salud de los equidos por producer una anemia y consecuentemente hipoxia que produce un deterioro multiorgánico. Se evaluó la presencia de AIE en los de equinos de trabajo de San Andrés Itzapa, Chimaltenango. Esto con el fin de evaluar la presencia de la enfermedad ya que este grupo de equinos forma parte de la seguridad alimentaria de la comunidad. Se analizaron 100 muestras de sangre utilizando la prueba de inmunodifusión en agar gel. Se encontró la presencia de la enfermedad en el $8 \%$ de los animales muestreados. La prevalencia fue mayor en hembras rrespecta los machos. La edad media de los equinos muestreados fue de 5 años. La condición corporal media de la población de estudio fue de 2. La presencia de la enfermedad en la población de estudio debe evaluarse y ser considerada como punto de partida para la implementación de medias de control y prevención dada la diseminación vectorial de la enfermedad y el impacto en la comunidad debido a la función zootécnia de los animales muestreados.

Palabras clave: anemia, equinos, inmunodifusión

Abstract- Equine infectious anemia (IEA) is a world wide distribution disease caused by a lentivirus associated with the presence of transmitting vectors of the gender Tabanus and Stomoxys. This disease has an impact on the health of the equines by producing anemia and consequently hypoxia that produces a multi-organ deterioration. The presence of AIE in the working equine of San Andrés Itzapa, Chimaltenango was evaluated. This in order to assess the presence of the disease since this group of equines is part of the food security of the community. 100 blood samples were analyzed using the gel agar immunodi-fusion test. The presence of the disease was found in $8 \%$ of the sampled animals. The prevalence was higher in females than in males. The mean age of the horses

Author a: Ejercicio profesional supervisado, Dirección de Extensión e investigación, Facultad de Medicina Veterinaria y Zootecnia, Universidad de San Carlos de Guatemala.

Author o: Departamento de Clínica de animales de compañía, Hospital Veterinario, Facultad de Medicina Veterinaria y Zootecnia, Universidad de San Carlos de Guatemala.

Author p: Departamento de Ayudas Diagnósticas, Hospital Veterinario, Facultad de Medicina Veterinaria y Zootecnia, Universidad de San Carlos de Guatemala.e-mail: danavilla47@gmail.com sampled was 5 years. The mean body condition of the study population was 2 . The presence of the disease in the study population should be evaluated and considered as a starting point for the implementation of control and prevention measures given the vector spread of the disease. -age and the impacton the community due to the zootechnical function of the sampled animals.

Keywords: anemia, equine, immunodiffusion.

\section{INTRODUCCIÓN}

os equinos son requeridos en las fincas con alta demanda del sector ganadero. En Guatemala, el grupo de ganado caballar tiene una función zootécnica de trabajo principalmente en áreas rurales. Además, forma parte de la seguridad alimentaria del hogar. En las montañas, el caballo mueve diversos productos en la agricultura, como el café y leña (Gamarro, 2019). Según el Instituto Nacional de Estadística de Guatemala (INE), en el último censo equino realizado en el 2003, se estima una población de 218,675 équidos.

La anemia infecciosa equina (AlE) es una enfermedad de distribución mundial que afecta a los équidos (Sánchez et al., 2018). Es causada por un vírus del género Lentivirus de la familia Retroviridae. Se ha reportado la presencia de la enfermedad en regiones con clima húmedo debido a la presencia de vectores transmisores del género Tabanus y Stomoxys calcitrans (Borges et. al., 2013).

La presentación clínica de la enfermedad suele ser crónica. Los animales infectados se convierten en portadores con signos inaparentes por lo que suele pasar inadvertida. Impacta negativamente tanto en la salud como el bienestar de los equinos, ocasionando pérdidas en el rendimiento y económicas en el ganado que es utilizado como fuerza de trabajo. (Garzón, 2015; Estrada, et al. 2018 y Sánchez, et al. 2018).

En Guatemala, el Ministerio de Agricultura, Ganadería y Alimentación (MAGA) registra proporciones de la enfermedad hasta de $18.54 \%$ entre los años 2005 al 2012. Por su parte Borja (2014) encontró $8.45 \%$ en un estudio retrospectivo sobre casos de AIE en Guatemala. 
Sin embargo, no todas las regiones del país han sido muestreadas en dichos estudios. En general, los equinos en Guatemala son una población poco estudiada, por lo que se desconoce el comportamiento epidemiológico de las enfermedades en el país. Borja (2014) menciona que avances relacionados a la salud equina del área en el año 2012 al 2013 son mínimos, y no se les brinda la atención necesaria lo que disminuye el tiempo de vida del equino.

San Andrés Itzapa es un municipio del Departamento de Chimaltenango cuya actividad económica depende del sector agrícola. La población estimada de équidos en el municipio de San Andrés Itzapa incluyendo sus aldeas y cantones es de 4,000 animales. Los équidos de trabajo en San Andrés Itzapa, representan una población aproximada de 1,100 (SABESA, 2019). Los equinos de San Andres Itzapa son ejemplares criollos en su mayoría. Son utilizados para el transporte en los caminos del área rural y trabajo de carga. Su alimentación se basa en el pastoreo, careciendo de suplementación y programas sanitarios (Mandrujano, 2017). Esto hace que muchos equinos sean utilizados como fuerza de trabajo, siendo un eje fundamental como colaborador en el trabajo diario para obtener el aporte económico para las familias.

El present estudio tiene la finalidad de encontrar la presencia de Anemia Infecciosa Equina en los equidos del municipio de San Andrés Itzapa, Chimaltenango. Esto con la finalidad de encontrar evidencia de la enfermedad y generar información respecto al comportamiento epidemiológico de la misma. De esta forma se podrán generar e implementar medidas de control y prevención en el área de estudio.

\section{Materiales y Métodos}

a) Área de estudio

El estudio se realizó en el municipio de San Andrés Itzapa, departamento de Chimaltenango; ubicado $65 \mathrm{~km}$ de la ciudad capital de Guatemala, cuyas coordenadas son $14^{\circ} 37^{\prime} 12^{\prime \prime} \mathrm{N}, 90^{\circ} 50^{\prime} 39^{\prime \prime} \mathrm{O}$.

\section{b) Criterios de inclusión}

En el estudio se incluyeron equinos de trabajo del municipio de San Andrés Itzapa, cuya función zootécnia fuera de trabajo (carga de material para posterior venta). Además, se incluyeron animales mayores a seis meses de edad, no importando el sexo y condición corporal.

\section{c) Tamaño de muestra}

El muestreo se realizó por conveniencia, en base a la previa autorización de los propietarios para participar en el estudio y colectar la muestra sanguínea de sus animales. De esta forma se obtuvo una muestra total de 100 equinos de trabajo.

\section{d) Toma de muestra}

Las muestras de sangre fueron obtenidas por punción de la vena yugular utilizando la técnica descrita por OIE (2005) y PAHO (2017). Se colectaron $10 \mathrm{ml}$ de sangre que fue colocada en tubos al vacío sin anticoagulante para su posterior extracción de suero. Las muestras fueron transportadas en hieleras, manteniendo una temperatura de $4^{\circ} \mathrm{C}$ hasta su llegada al Laboratorio de Sanidad Animal del VISAR del Ministerio de agricultura y Ganadería de la ciudad de Guatemala, en un periodo no mayor de 24 horas.

Se tomaron registros de los animales muestreados como sexo, edad, peso y condición corporal según escala de Carroll \& Huntington (1988).

\section{e) Prueba diagnóstica}

La prueba utilizada para la identificación de la Anemia Infecciosa Equina en los équidos fue inmunodifusión en agar gel según el protocolo indicado por la Organización mundial de sanidad animal (OIE, 2019).

\section{f) Análisis de datos}

Los datos obtenidos fueron resumidos utilizando estadística descriptiva y tablas de contingencia, utilizando distribuciones de frecuencias, medias para las variables.

\section{ili. Resultados}

Se encontró la presencia de Anemia Infecciosa Equina en la población de estudio utilizando la prueba de inmunodifusión en agar gel. La prevalencia de los animales muestreados fue del $8 \%$. En el cuadro 1 se describen las prevalencias por sexo.

Cuadro 1: Distribución de frecuencias de equinos muestreados para Anemia infecciosa equina

\begin{tabular}{|c|c|c|c|c|c|}
\hline \multirow{2}{*}{ Sexo } & \multicolumn{2}{|c|}{ Positivos } & \multicolumn{2}{c|}{ Negativos } & \multirow{2}{*}{ Total } \\
\cline { 2 - 5 } & $N$ & $\%$ & $N$ & $\%$ & \\
\hline Hembras & 5 & 10 & 50 & 90 & 55 \\
\hline Machos & 3 & 6.67 & 42 & 93.3 & 45 \\
\hline
\end{tabular}

La edad media de los equinos muestreados fue de 5 años.

En la Cuadro 2 se presenta la categorización

de las muestreados. 
Cuadro 2: Categorización de la edad de los equinos muestreados a AlE

\begin{tabular}{|c|c|c|c|c|c|}
\hline \multirow{2}{*}{ Edad } & \multicolumn{2}{|c|}{ Positivos } & \multicolumn{2}{c|}{ Negativos } & \multirow{2}{*}{ Total } \\
\cline { 2 - 5 } & $N$ & $\%$ & $N$ & $\%$ & \multirow{2}{*}{35} \\
\hline 6 meses a 2 años & 1 & 2.86 & 34 & 97.14 & 35 \\
\hline 3 a 6 años & 2 & 5.71 & 33 & 94.29 & 13 \\
\hline 7 a 9 años & 1 & 7.69 & 12 & 92.31 & 17 \\
\hline Más de 10 años & 4 & 23.53 & 13 & 76.47 & \\
\hline
\end{tabular}

La condición corporal de la población de estudio fue de 2. En el cuadro 3 se presenta la condición corporal de los animales muestreados utilizando la escala de Carroll \& Huntington (1988).

Cuadro 3: Condición corporal de los equinos muestreados.

\begin{tabular}{|c|c|c|c|c|c|}
\hline \multirow{2}{*}{$\begin{array}{c}\text { Condición } \\
\text { corporal }\end{array}$} & \multicolumn{2}{|c|}{ Positivos } & \multicolumn{2}{c|}{ Negativos } & \multirow{2}{*}{ Total } \\
\cline { 2 - 5 } & $N$ & $\%$ & $N$ & $\%$ & \\
\hline 1 & 2 & 13.33 & 13 & 86.67 & 15 \\
\hline 2 & 6 & 7.59 & 73 & 92.41 & 79 \\
\hline 3 & 0 & 0 & 6 & 6 & 6 \\
\hline 4 & 0 & 0 & 0 & 0 & 0 \\
\hline 5 & 0 & 0 & 0 & 0 & 0 \\
\hline
\end{tabular}

\section{Discusión}

La presencia de AlE en los equinos muestreados puede deberse a las condiciones climáticas del área de estudio. El municipio de San Andrés Itzapa es una zona de bosque húmedo tropical, las cuales son condiciones ambientales que permiten la distribución del vector. Según Craigo \& Montelaro (2008) la incidencia de caballos infectados con AIE es la más alta en climas tropicales y subtropicales. Por lo tanto, el clima tropical y la presencia de humedales es favorable para el crecimiento de vectores mecánicos y la carga de los mismos puede ser mayor que en otros climas permitiendo así la constante exposición de équidos susceptibles a vectores hematófagos y équidos infectados (Borges et al., 2013). Además, debe de considerarse la transmisión puede ser iatrogénica por el mal uso de agujas o instrumentos contaminados y a través del calostro (Clabough, 1993).

En cuanto a la edad, la media de los equinos muestreados fue de 5 años siendo el 23.53\% equinos mayores de 10 años. Borges et al. (2013), indica que los equinos de trabajo y equinos mayores de 10 años aumentan su probabilidad de exposición por vectores y a cualquier otro tipo de transmission iatrogénica de la enfermedad.

La condición corporal de la población de estudio fue de 2 , siendo un $7.59 \%$ corresponde a condición corporal 2 y un 13.33 en condición corporal 1 .
Algunos factores externos relacionados a la finalidad laboral, horas de trabajo, alimentación y salud pueden ser reflejados en la condición.

Las prevalencias obtenidas en el presente estudio, son similares a los resultados obtenidos por Borga (2014). Sin embargo, difieren con los estudios realizados por el MAGA en los años 2005 al 2012. Esto puede deberse al tamaño de la muestra, las áreas y la amplitud del estudio. Garzón (2015) hace referencia a estudios que indican la prevalencia de AIE en América Latina que oscilan desde el $2 \%$ al $28 \%$ donde se evidencia la falta de investigación de la enfermedad principalmente en Centro América. Sin embargo, Centroamérica puede tener resultados similares a Nicaragua (8.07\%) como sugiere Garcia (2015). Esto se debe a que en esta reigón predominan las zonas tropicales o subtropicales donde permite una fácil diseminación del virus por medio de los vectores mecánicos favorecidos por las condiciones climáticas y geográficas (Estrada et al, 2018; Sánchez et al, 2018; Garzón, 2015; García, 2015 y Morales, 2015). Por esta razón, es necesario llevar a cabo más investigaciones de la distribución de la enfermedad. 


\section{Agradecimientos}

A los supervisores por las valiosas sugerencias. A los propietarios que permitieron las tomas de muestras en sus animales. A SABESA, MV Leonardo Montufar, Lic. Alejandro Salazar, Alejandra de Matta, René Porras y Wilson Azurdia por el material, transporte y apoyo brindado para la toma de muestras. A la Dra. Evelyn Godoy y al Laboratorio de Sanidad Animal del VISAR del Ministerio de Agricultura y Ganadería por las pruebas.

\section{References Références Referencias}

1. Borja, J. (2014) Estudio retrospectivo sobre casos de Anemia Infecciosa Equina en Guatemala durante los años 2011 y 2012, tomando como base las muestras procesadas en el laboratorio de microbiología de la Facultad de Medicina Veterinaria y Zootecnia. Recuperado de: http://ww w.repositorio.usac.edu.gt/1866/2/Tesis\%20Med\%20 Vet\%20Julio\%20Borja.pdf

2. Borges, A., Silva, L., Nogueira, M., Oliveira, A., Segui, N., Ferreira, F., Witter, R. \& Aguilar, D. (2013) Prevalence and risk factors for Equine Infectious Anemia in Poconé municipality, northern Brazilian Pantanal. Recuperado de: https://www.sciencedirec t.com/science/article/abs/pii/S0034528813000659

3. Camino, E., \& Cruz, F. (2017) AlE: Anemia Infecciosa Equina. Centro de Vigilancia Sanitaria Veterinaria (VISAVET). Recuperado de: https://www.visavet.es/es/articulos/aie-anemia-infec ciosa-equina.php

4. Carroll, C. \& Huntington P. (1988) Body condition scoring and weight estimation of horses. Equine Veterinary Journal. 20(1), 41-45. doi:10.1111/j.20423306.1988.tb01451.x

5. Clabough, D. (1993) Equine Infectious Anemia. Veterinaryclinics of North America: Equine Practice. 321-336. Recuperado de: https://www.sciencedir ect.com/science/article/abs/pii/S0749073917303991

6. Craigo, J. \& Montelaro, R. (2008) Equine Infectious Anemia Virus. Recuperado de: https://www.sci encedirect.com/science/article/pii/B9780123744104 003952

7. Estrada, A., Alva, M., Muñoz, S., Ramirez, E., Canales, M., López, A., \& Galván, R. (2018) Seroprevalencia de Anemia Infecciosa Equina en Équidos del Estado de Veracruz, México. Research Gate. Recuperado de: https://www.researchgate.net/publication/3295896 22 SEROPREVALENCIA_DE_ANEMIA_INFECCIOS A EQUINA EN EQUIDOS DEL ESTADDO DE VER

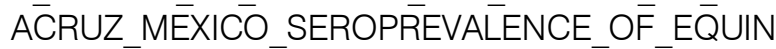
E_INFECTIIOUS_ANNEMIA_IN_EQUINES_AT_THE_S TATTE_OF_VERACRUZ_MEXICO

8. Gamarro, U. (2019) Mercado exclusivo y atractivo. Reportaje de Prensa Libre. Recuperado de: https://www.pressreader.com/guatemala/prensa-lib re/20190811/281736976084697

9. García, M. (2015) Seroprevalencia de Anemia Infecciosa Equina en caballos Pura Raza Española, del Municipio de Chinandega, durante los meses de Enero - Junio del 2015. Recuperado de: http://riul.unanleon.edu.ni:8080/jspui/bitstream/1234 56789/4090/1/229275.pdf

10. Garzón, M. (2015) Estado actual de la Anemia Infecciosa Equina en Colombia y América Latina. Recuperado de: https://repository.javeriana.edu .co/bitstream/handle/10554/16669/GarzonFlorezMo nicaRocio2015. pdf?sequence $=1$ \&isAllowed $=y$

11. Mandrujano, A. (2017) Estudio comparativo del efecto del uso de semilla de papaya, en dos diferentes intervalos de administración, sobre la cantidad de huevos por gramo de heces, en equinos de San Andrés Itzapa, Chimaltenango. Recuperado de: http://www.repositorio.usac.edu. gt/7731/1/Tesis\%20Med\%20Vet\%20Ana\%20Gabriel a\%20Mandujano.pdf

12. Manual Terrestre de la OIE (2019) Anemia Infecciosa Equina. Capitulo 3.5.6. Recuperado de: https://www.oie.int/fileadmin/Home/esp/Health_stan dards/tahm/3.05.06 EIA.pdf

13. Manual Terrestre de la OIE (2005) Capítulos introductorios. Sección 1. Capitulo 1.1.1. Recuperado de: https://www.oie.int/fileadmin/Hom e/esp/Animal_Health_in_the_World/docs/pdf/1.1.01 Muestreo_2007.pdf

14. Morales, A., Sánchez, A., \& Morales, M. (2015) Anemia Infecciosa Equina. Una Revisión. NHRR vol.46 no.1-2 Caracas. Recuperado de: http://ve.scielo.org/scielo.php?script=sci_arttext\&pi $d=$ S0798-04772015000100008

15. PAHO (2017) Manual veterinario de toma y envío de muestras. Manual técnico. Recuperado de: https://iris.paho.org/handle/10665.2/34527

16. SABESA (2019) Lugares de enfoque, redes de bienestar equino. SABESA. Guatemala.

17. Sánchez, A., Estrada, A., Alva, M., Munoz, S., López, A. \& Canales, M. (2018) Diagnóstico serológico de Anemia Infecciosa Equina y Piroplasmosis en équidos de trabajo del municipio de Veracruz, Veracruz, México. Recuperado de: http://www.colpos.mx/agrocien/Bimestral/2018/dicespecial/art-5.pdf 